\title{
AGLIETTA E A TEORIA DA REGULAÇÃO: DIREITO E CAPITALISMO
}

\author{
Tese de Doutorado \\ Orientador: José Maria Arruda de Andrade \\ UNIVERSIDADE DE SÃO PAULO \\ FACULDADE DE DIREITO
}




\section{ALESSANDRA DEVULSKY DA SILVA TISESCU}

Aglietta e a Teoria da Regulação: Direito e Capitalismo

Tese apresentada à Faculdade de Direito da Universidade de São Paulo para obtenção do título de Doutor em Direito.

Área de Concentração: Direito Econômico e Financeiro.

Orientador: Prof. Dr. José Maria Arruda de Andrade

São Paulo 


\section{RESUMO}

Para compreender a dinâmica pela qual o regime de acumulação e os modos de regulação se articulam, submetidos à luta de classes, Michel Aglietta teve por objetivo primordial dedicar-se aos estudos desse fenômeno. A perspectiva teórica da escola parisiense da regulação de Aglietta inicia-se ela análise da crise dos Estados Unidos, por meio da qual se estabelece o processo de acumulação profundamente ligado à taxa de mais-valia do modelo fordista, o qual chega às práticas do Estado, bem como aos seus limites. Os fundamentos teóricos, estabelecidos nas primeiras três partes desta tese, objetivam apresentar um contexto cujos temas e discussões serão desenvolvidas nos próximos capítulos, que precisam dessa abordagem preliminar de maneira a estabelecer um encadeamento lógico. Por essa razão, efetuar-se-á a análise das raízes marxistas nas proposições teóricas de Aglietta, que surgem do esforço de se conceitualizar ideias que correspondam à representação do movimento histórico real, sob a ótica de uma significação social, vinculada ao materialismo dialético. Assim, considera-se que a regulação do capitalismo tem origem na criação social, na qual o processo antagônico fundamental - inerente à relação salarial - é a força motriz da acumulação do capital. Daí, pode-se afirmar que o processo de concentração e centralização do capital é mediado pelo Estado. Essa proposição regulacionista sofre forte influência do althusserianismo, uma vez que os conceitos de reprodução e de estruturas são utilizados no desenvolvimento dos, também, conceitos de regulação e de modo de regulação. Na conclusão deste trabalho, analisa-se o papel do Estado, a partir da teoria da regulação, em especial, como instrumento do modo de regulação, a fim de se compreender as mediações realizadas sobre as crises, a relação salarial e a política econômica - instâncias estas em profunda articulação com o Direito.

Palavras-chave: Michel Aglietta, Teoria da regulação, Marxismo, Capitalismo, Direito. 


\begin{abstract}
The need to understand the dynamics by which the regime of accumulation and modes of regulation are based, subject to the class struggle, is the main purpose of the research of Michel Aglietta. The theoretical point of view of the Parisian school of regulation orchestrated by Aglietta begins with the analysis of the crisis in the U.S., established on the accumulation process and deeply linked to the rate of surplus value of the fordist model, arriving in the practices of the State, though also its limits. The theoretical foundations established in the first three parts of this thesis aims to provide a context to the themes and discussions that will be developed in subsequent chapters, which need this preliminary approach to your own connectedness. For this reason, we conduct the analysis of Marxist roots within the theoretical propositions of Aglietta, proposals emerging in the effort to design concepts to match the representation of the historical real, social significance, by dialectical materialism. Whereas the regulation of capitalism is a social setting, in which the fundamental process underlying the antagonistic wage relationship is the driving force of capital accumulation, it can be said that the process of concentration and centralization of capital is mediated by the State. This regulationist's proposition has in the Althusserianism a strong influence, since the playback concepts and structures are used in development of the concepts of control and regulation mode. Going into the final part of the analyzes, the role of the State rest established by regulation theory, in particular, as a tool of the mode of regulation in order to understand the mediations conducted on the crisis, the wage relation and economic policy - wich in these instances have deep connection with the law.
\end{abstract}

Keywords: Michel Aglietta, Regulation Theory, Marxism, Capitalism, Law. 


\section{RÉSUMÉ}

Le besoin de comprendre la dynamique par laquelle le régime d'accumulation et des modes de régulation s'articulent, subordonnés à la lutte des classes, est le but de la recherche principale de Michel Aglietta. Le point de vue théorique de l'école parisienne de la régulation orchestré par Aglietta commence avec l'analyse de la crise aux Etats-Unis, établi sur les processus d'accumulation et profondément lié au taux de plus-value du modèle fordiste, arrivant aux pratiques de l'État, bien aussi de ses limites. Les fondements théoriques mis en place dans les trois premières parties de cette thèse ont l'intention de donner un contexte au thème et les discussions menées dans les chapitres postérieurs ont besoin de cette approche préliminaire pour son propre enchainement logique. Pour cette raison, nous effectuerons l'analyse des racines marxistes présentes dans les propositions théoriques d'Aglietta, propositions émergentes dans l'effort de concevoir des concepts capables de correspondre à la représentation du mouvement historique réel, ayant une signification sociale, par le matérialisme dialectique. Considérant que la réglementation du capitalisme est un création social, dans lequel le processus fondamental qui sous-tend la relation salariale antagoniste est la force motrice de l'accumulation du capital, on peut dire que le processus de concentration et de centralisation du capital est médiée par l'Etat. Cette proposition régulationniste a une forte influence de l'althussérianisme, puisque les concepts de reproduction et de structures sont utilisées dans le développement des concepts de contrôle et le mode de règlement. En s'adressant à la partie finale de la thèse, le rôle de l'État établi par la théorie de la régulation est analisée, en particulier, comme un outil de la mode de régulation afin de comprendre les médiations menées sur la crise, le rapport salarial et de la politique économique - dans ces cas avec une connexion profonde avec le Droit.

Mots-Clés: Michel Aglietta, Théorie de la Régulation, Marxisme, Capitalisme, Droit. 


\section{INTRODUÇÃO}

A teoria da regulação tem sido objeto de estudo de muitas pesquisas, e uma das primeiras foi a de Michel Aglietta em 1976 - Régulation et crises du capitalisme -, que iniciou o que se poderia denominar como um "ramo" investigativo em inúmeras escolas marxistas associadas à teoria da política econômica. A relação salarial, o impacto das novas tecnologias, o regime de acumulação, o modo de regulação e a tendência às crises são alguns dos objetos de investigação nos estudos de Aglietta. Com alguma variação, muitos conceitos foram revisitados e incorporados aos trabalhos da escola parisiense da regulação, na qual se destacaram, também, como um marco metodológico: Robert Boyer e Alain Lipietz, dos quais muitas de suas contribuições serão incorporadas a esta pesquisa, sempre que estabelecerem uma relação de fidedignidade com o eixo teórico de Aglietta, o qual será a principal fonte deste trabalho.

É factível afirmar que, a teoria da regulação foi enriquecida, posteriormente, por diversos trabalhos na França, que criaram três grupos distintos dentro da chamada teoria da regulação: a escola fundada por Paul Boccara, também, denominada por boccarien, ou boccariana. Tal origem ocorre quando o seu fundador era o chefe econômico do partido comunista francês, que direcionava o campo de atuação do grupo à análise da dinâmica das mediações realizadas pelas crises. Além disso, a escola grenobloise (formada pelo "Grupo de pesquisas sobre a regulação das economias capitalistas" - GREEC) envereda pela pesquisa das variações das taxas de lucros e o seu desenvolvimento em termos concorrenciais - a reunir uma gama bastante diversificada de teóricos a exemplo de Gerbier, Vigezzi, Del Forno, Di Ruzza Calvet. Entre eles destacaram-se Gérard Destanne de Bernis e Rolande Borrelly por causa da maior difusão de suas pesquisas acerca do caráter transnacional do capital, em que importa menos a figura do Estado-nação do que o sistema produtivo. O Estado-nação integra-se a esse sistema, notadamente, por ser o conjunto plurinacional composto por um Estado-matriz e Estados-filiados ao elemento determinante da política econômica a ser adotada. Assim, a escola parisiense se tornou a mais reconhecida entre as demais e tem, até hoje, Aglietta como o seu principal representante. 
Neste trabalho, desconsiderar-se-ão as escolas da Alemanha Ocidental, a de Amsterdã, conhecida como nórdica e a dos americanos radicais que, apesar de terem importantes pesquisas sobre a teoria da regulação, não se detiveram à questão da relação salarial, pois algumas propostas se afastaram do trabalho inicial de Aglietta. Dessa forma, este estudo tem por objetivo compreender os principais conceitos desenvolvidos por Aglietta que, mesmo não inspirado nos conceitos marxistas, estabelecem uma relação muito próxima à teoria política econômica de Marx, o que revela assim uma aversão aos princípios da teoria neoclássica econômica ou ortodoxa.

A teoria da regulação parisiense parece adotar o materialismo histórico como premissa fundamental de suas pesquisas. Esses estudos de Aglietta têm o mérito de demonstrar que, as crises experimentadas nas diversas fases do capitalismo, não são frutos de um mecanismo de "autorregulação" do mercado, mas apresentam uma intrincada rede de fatores que regem as suas eclosões. As crises estão diretamente relacionadas com o regime de acumulação e o modo de regulação, o que determina ser de fundamental importância que a historicidade do seu desenvolvimento faça parte dos elementos de pesquisa a ser adotada.

Aglietta adota a experiência capitalista norte-americana como modelo de desenvolvimento, a funcionar ora como um "museu" do desenvolvimento do capitalismo moderno, ora como uma plataforma privilegiada de investigação dos futuros delineamentos da política econômica neoliberal. Nesse aspecto, os modelos fordista e taylorista no processo produtivo serão de suma importância para a compreensão das consequências geradas pela integração de novas tecnologias e de novas maneiras de organização do trabalho. Diante desse quadro e, especialmente, por meio das investigações do desenvolvimento capitalista no período que compreende o final do século XIX até as décadas de 1960 e 1970, do século XX, Aglietta propõe - apesar de outros conceitos atinentes ao processo de acumulação o conceito de modo de regulação.

O modo de regulação compreende um aparato de mecanismos e de instituições responsável por auxiliar, dentro da dinâmica do capitalismo moderno, a equalização do comportamento do Departamento I e o do Departamento II, que naturalmente tendem a desequilíbrios. Esse fenômeno leva à queda da taxa da maisvalia que, dentro do processo de acumulação, segundo Aglietta, é um dos fatores 
desencadeante das crises. Dessa forma, o modo de regulação está intimamente ligado ao sucesso, ou ao fracasso de uma política econômica que ensaie evitar a chamada crise estrutural, no entanto, o modo de regulação não se restringe ao controle do câmbio, da inflação, da política monetária e financeira. As políticas públicas também fazem parte desse processo como um importante eixo necessário à manutenção da estabilidade do regime de acumulação, na qual o Estado se apresenta como protagonista.

Portanto, compreender de que modo a figura do Estado compõe o cenário regulatório proposto por Aglietta; resultante de sua pesquisa inaugural sobre as crises, tem relevância vital, pois as relações salariais e as alterações do regime de acumulação, permitiriam também compreender de que modo o modelo brasileiro de Estado é construído, e de que maneira a economia periférica brasileira é impactada pela economia líder norte-americana. O resultado da interação entre os regimes de acumulação e os modelos de regulação molda as instituições, e a hipótese é a de que, no caso brasileiro, não se distancia desse processo. Para a compreensão estrutural das causas que constituem a moderna figura do Estado associada ao capitalismo - segundo os pressupostos de Aglietta e da escola parisiense de regulação - isso seria válido para se entender as fragilidades e as virtudes do Estado moderno brasileiro, guardadas as especificidades dos fenômenos políticos e econômicos latino-americanos.

Desse modo, o primeiro capítulo estabelece os fundamentos primordiais da teoria da regulação, especialmente aqueles ligados a Aglietta e à escola parisiense. Neste capítulo, estabelecer-se-ão as possíveis relações entre o marco teórico da regulação, além do materialismo histórico e dialético de Marx, tendo-se por foco o momento em que Louis Althusser, um dos principais intermediários, relaciona os conceitos de reprodução e de regulação com os da análise da infraestrutura e superestrutura.

No segundo capítulo, serão apresentados os conceitos de regime de acumulação e modo de regulação, sobre os quais se erige toda a construção teórica regulacionista de Aglietta, notadamente, por meio da experiência norte-americana. A estabilidade e a decadência do regime de acumulação eleito aparecem atreladas à taxa de mais-valia, por meio de um movimento, no qual a relação salarial surge para desempenhar papel importante na regulação desses elementos. A rigidez ou a 
flexibilidade na fixação de salários, desde o modo de organização impresso no trabalho até aquele cujas negociações coletivas são engendradas pelos sindicatos de ambas as esferas laborais. Isso permite haver fatores que podem ser utilizados na identificação da proximidade de uma crise, seja para compreender as suas causas materiais, seja para evitá-las.

O terceiro capítulo terá como foco as principais distinções entre a perspectiva regulacionista de Aglietta e a escola neoclássica, a fim de se estabelecer o tratamento adequado aos fenômenos econômicos estudados. A concepção de moeda e a análise do seu surgimento até a modernidade serão tratadas a partir de uma breve perspectiva histórica, incluindo-se o seu desenvolvimento e a categoria de instituição. A concorrência, além dos fenômenos da concentração e de centralização do capital, incluindo-se a sua modalidade financeira, serão tratados de modo a demonstrar a sua relação com a mudança no padrão de financiamento da produção. Junto a esse processo de centralização, o desenvolvimento de fundos de pensão também é estudado concomitantemente à fixação do salário nominal e aos seus efeitos sobre a vida do trabalhador e ao processo de acumulação do capital. Além disso, a financeirização do capital é apontada como uma das características do desenvolvimento capitalista pós-fordista, indicando-se como um entre os vários efeitos, a extrema estratificação da classe capitalista.

No quarto e último capítulo, a figura do Estado é analisada sob a perspectiva do autor, de maneira a elaborar um possível conceito da forma Estado e de sua articulação com os mecanismos capitalistas, de acordo com critérios propostos pelo modo de regulação. A relação entre o Estado e a economia é apresentada sob parâmetros marxianos, os quais permitem verificar que as relações de produção influenciam de modo importante a natureza das suas ações interventivas. Desse modo, os campos de atuação estatal e a política econômica adotada mostram certa ambiguidade em sua articulação com a luta de classes, por vezes abrindo-se possibilidade de transformações da relação salarial ou de manutenção de um regime de acumulação, assim como na sua atuação diante de crises econômicas. As intervenções estatais junto à economia são tratadas como uma necessidade da própria constituição do mecanismo da produção capitalista, o que diferencia a teoria da regulação das teorias neoliberais no que tange à compreensão dessa relação. 


\section{CONCLUSÃO}

A teoria desenvolvida por Aglietta em Régulation et Crises du capitalisme inova a compreensão do capitalismo atual em três novas frentes: a da natureza das crises do capitalismo; a da articulação entre regimes de acumulação e modos de regulação, incluindo-se a da relação salarial. Ainda que o tratamento conceitual a cada uma delas seja específico - cuja qualidade é não repetir os erros cometidos pelas teorias neoclássicas - essas instâncias estão articuladas na realidade e são também estudadas em conjunto.

Vale acrescentar que, por meio de uma análise que enfatiza a historicidade dos objetos da pesquisa - cujo método se aproxima do materialismo-dialético marxiano - o autor revisita alguns conceitos da tradição econômica, atribuindo-lhes um novo sentido para aprimorá-los, ou simplesmente abandonar aqueles que são inadequados à compreensão do capitalismo moderno. Muito embora os fenômenos estudados refiram-se ao desenvolvimento do fordismo e pós-fordismo nos Estados Unidos durante o século XX, é possível traçar um paralelo entre as conclusões do autor e as realidades distintas que se construíram ao longo do tempo.

No que concerne às crises que solapam as economias de centro e da periferia com causas, ritmos e prolongamentos diversos, verifica-se que elas eclodem por força do antagonismo inerente à forma do capital. Em virtude disso, elas são cíclicas e causadas pelas inconstâncias de um regime de acumulação, ou mesmo pelas falhas dos instrumentos de mediação, que se agregam ao modo de regulação estabelecida. No entanto, as crises causadas pela alternância do regime de acumulação ou mesmo pelas falhas dos instrumentos de mediação integrados ao modo de regulação estabelecida, as crises possibilitam transformações no processo de acumulação que reverberam nos modos de regulação.

Ao alçar o fordismo à posição de modelo privilegiado de um modo de regulação, Aglietta percebe que não é possível aplicar o instrumental teórico ortodoxo à análise econômica sem comprometer o entendimento da totalidade dos fenômenos. A dualidade entre equilíbrio e desequilíbrio é abandonada e será substituída pela dialética entre regulação e crise. 
Cabe dizer que com as crises sistêmicas afetando as relações salariais, cuja articulação com as outras instâncias econômicas é capaz de perturbar de um lado, ou estabilizar, de outro, as taxas de lucro, o desafio é encontrar em Régulation et Crises du capitalisme o papel do Estado como instância de mediação. O conceito de Estado no âmbito do processo de regulação é encontrado na obra inaugural da escola teórica, mas de forma esparsa e sem a sistematização que poderia tornar mais claro o sentido proposto pelo autor à estrutura estatal.

Em obras remanescentes a figura do Estado também chega a ser tratada, mas sutilmente e desvincula-se gradualmente dos principais elementos que aproximavam à abordagem do conceito aos parâmetros metodológicos marxianos, o que especialmente também ocorre com o direito.

Nota-se que a forma-Estado surge então como estrutura vinculada ao modo de regulação, à qual se atribui uma posição estratégica de sustentação do capitalismo. Aglietta reconhece a importância das intervenções estatais no arrefecimento das crises e no estabelecimento de políticas públicas capazes de servir como mediadores de sua prevenção.

Ressalta-se que o desafio do emprego da figura do Estado na promoção de direito sociais, e no estabelecimento de políticas públicas orientadoras do desenvolvimento econômico, é reconhecer que, conforme pensadores marxistas como Althusser, Poulantzas e Gramsci - fontes inspiradoras de Aglietta -, as contradições do capital acompanham a dinâmica do Estado. Desse modo, ainda que o Estado tenha sido atingido pelos antagonismos originados nas lutas de casse, que permeiam a sociedade civil e a sociedade política, o capital se afigura como o principal beneficiário de suas medidas intervencionistas.

Ainda que Aglietta tenha gradativamente substituído a dimensão da luta de classes pela da relação salarial, e que isso restrinja a amplitude das ações do Estado, as melhorias das condições dos trabalhadores ocorre, mesmo de maneira ínfima direta ou indiretamente, por intermédio daquela instituição. Assim, o encanto de Aglietta pelo Estado salarial aproxima-o de uma posição reformista que não se coaduna com aquela composição conceitual de seus tratados iniciais.

Portanto, a transformação emancipatória a que se pretende viabilizar por meio de instrumentos regulatórios não ocorre exclusivamente nas instâncias do aparato estatal, muito embora, isso tenha importância vital na dinâmica das relações 
de produção. Diante disso, somente o conhecimento das limitações existentes na relação entre Estado e capital possibilita catalisar as funcionalidades interventivas a favor do desenvolvimento social que, a longo prazo, podem influenciar a luta de classe e impulsionar a sociedade a um novo horizonte. 


\section{REFERÊNCIAS}

AGLIETTA, Michel. A theory of capitalist regulation: the US experience. London: Verso, 2000.

. Régulation et crises du capitalisme. Paris: Odile Jacob, 1997.

. Regulación y crisis del capitalismo : la experiencia de los Estados Unidos. Madrid: Siglo veintiuno, 1979.

La crise: comment en est-on arrivé là ? comment en sortir ?. Paris : Michalon, 2008.

La crise: les voies de sortie. Paris : Michalon Éditions, 2010.

La fin des devises clés. Paris: La découverte, 1986.

Macroeconomia financeira: mercado financeiro, crescimento e ciclos. V. 1. São Paulo: Loyola, 2004.

Macroeconomia financeira: crises financeiras e regulação monetária. V. 2. São Paulo: Loyola, 2004.

Phases of US capitalist expansion. New Left Review, n. 110, p. 17-28. Jul.-Aug. 1978.

.Sobre algunos aspectos de las crisis en el capitalismo contemporáneo. In: Ontiveros, E. Rupturas de un sistema económico. Madrid: H. Blumes, 1981. 
; BRENDER, Anton. Les métamorphoses de la société salariale : la France en projet. Paris : Calmann-Lévy, 1984.

; KHANNICHE, Sabrina; RIGOT, Sandra. Les hedge funds: entrepreneurs ou requins de la finance. Paris: Perrin, 2010. 2007.

; LANDRY, Yves. La Chine vers la superpuissance. Paris : Economica, ; MOATTI, Sandra. Le FMI: de l'ordre monétaire aux désordres financiers. Paris : Economica, 2000.

; ORLÉAN, André. A violência da moeda. São Paulo: Brasiliense, 1990. 2009. ; RIGOT, Sandra. Crise et rénovation de la finance. Paris : Odile Jacob, ALMEIDA FILHO, Niemeyer; PAULANI, Leda Maria. Regulação social e acumulação por espoliação: reflexão sobre a essencialidade das teses da financeirização e da natureza do estado na caracterização do capitalismo contemporâneo. Econ. Soc., Campinas, v. 20, n. 2, p. 243-72, Aug. 2011.

ALTHUSSER, Louis. A favor de Marx. 2. ed. Rio de Janeiro: Zahar, 1972.

A transformação da filosofia seguido de Marx e Lénine perante Hegel. Lisboa: Editorial Estampa, 1981.

Análise crítica da teoria marxista. Rio de Janeiro: Zahar, 1967. 
. Aparelhos ideológicos de estado. 9. ed. São Paulo: Graal, 2003.

Écrits philosophiques et politiques. Paris: Stock/Imec, 1994.

. Éléments d'auto-critique. Paris: Hachette, 1974.

. Freud e Lacan, Marx e Freud. 4. ed. Rio de Janeiro: Graal, 2000.

. Lênin e a filosofia. São Paulo: Mandacaru, 1989.

. Marxismo segundo Althusser. São Paulo: Sinal, [19..]. (Coleção sinal, 2).

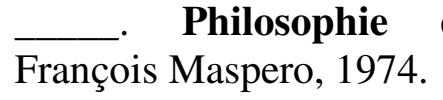

. Posições I. Rio de Janeiro: Graal, 1978.

. Posições II. Rio de Janeiro: Graal, 1980.

. Réponse a John Lewis. Paris: François Maspero, 1973.

. Sobre a reprodução. Petrópolis: Vozes, 1999.

. Solitude de Machiavel. Paris: Presses universitaires de France, 1988.

22 éme congrés. Paris : François Maspero, 1977. 
; BALIBAR, Étienne; ESTABLET, Roger. Ler o capital. Rio de Janeiro: Zahar, 1980. v. 2.

; __ _ _ L _ Lire le capital. Paris: François Maspero, 1967. v. 2.

; RANCIÈRE, Jacques; MACHEREY, Pierre. Ler o capital. Rio de Janeiro: Zahar, 1979. v. 1.

; ___ _ L L _ Lire le capital. Paris: François Maspero, 1967. v. 1.

et al. Da ideologia. Rio de Janeiro: Zahar, 1980.

et al. Dialética e ciências sociais. Rio de Janeiro: Zahar, 1967.

. et al. Epistemologia 2. São Paulo: Tempo Brasileiro, 1973.

ANDERSON, Perry. Considerações sobre o marxismo ocidental: nas trilhas do materialismo histórico. São Paulo: Boitempo, 2004.

ANDRADE, José Maria Arruda de Andrade. Do texto à norma e da norma ao texto: o aspecto construtivo da aplicação jurídica. In: Direito tributário: homenagem a Paulo de Barros Carvalho. São Paulo: Quartier Latin, 2006.

ANDRADE, José Maria Arruda de. Economicização do direito concorrencial. São Paulo: Quartier Latin, 2014. p. 30). 
ARANTES, Paulo. Extinção. São Paulo: Boitempo, 2007.

1964: o ano que não terminou. In: SAFATLE, V.; TELES, E. (Orgs). O que resta da ditadura: a exceção brasileira. São Paulo: Boitempo, 2010. p. 206).

ARRIGHI, Giovanni. O longo século XX: dinheiro, poder e as origens de nosso tempo. Rio de Janeiro: Contraponto, 2012.

BADIOU, Alain. Petit panthéon portatif. Paris: La fabrique, 2008.

BERCOVICI, Gilberto. Constituição econômica e desenvolvimento: uma leitura a partir da constituição de 1988. São Paulo: Malheiros, 2005.

Constituição e Estado de Exceção Permanente. Rio de Janeiro: Azougue Editorial, 2004.

. Desigualdades regionais, estado e constituição. São Paulo: Max Limonad, 2003.

. O ainda indispensável direito econômico. In: BENEVIDES, Maria Victoria de Mesquita; BERCOVICI, Gilberto; MELO, Claudineu de. (Org.). Direitos humanos, democracia e república: homenagem a Fabio Konder Comparato. São Paulo: Quartier Latin, 2009.

Planejamento e políticas públicas: por uma nova compreensão do papel do estado. In: BUCCI, Maria Paula Dallari (Org). Políticas públicas: reflexões sobre o conceito jurídico. São Paulo: Saraiva, 2006.

BOYER, Robert. A teoria da regulação: uma análise crítica. São Paulo: Nobel, 1990. p. 43. 
. Avant-propos à la seconde édition. In: BOYER, R.; SAILLARD, Y. (Org). Théorie de la régulation: l'état des savoirs. Paris: La Découvert, 2002.

Teoria da regulação: os fundamentos. São Paulo: Estação Liberdade, 2009.

BRAGA, Ruy. A nostalgia do fordismo: modernização e crise na teoria da sociedade salarial. São Paulo: Xamã, 2003. P. 77.

A vingança de Braverman ou a 'outra face' do trabalho informacional. Revista de Instituto de Estudos Socialistas, São Paulo: n. 15, p. 121-150, out. 2007.

BRENNER, Robert; GLICK, Mark. The regulation approach: theory and history. In: JESSOP, B. Regulation theory and the crisis of capitalism: the parisian regulation school. Cheltenham: Edward Elgar, 2001. p. 341.

BRUNHOFF, Suzanne de. A moeda em Marx. Rio de Janeiro: Paz e Terra, 1978.

A política monetária: um ensaio de interpretação marxista. Rio de Janeiro: Paz e Terra, 1978.

Crise capitalista e política econômica. In: Poulantzas, N. (Org). O estado em crise. Rio de Janeiro: Graal, 1985.

Finança, capital, estados. In: Brunhoff, Suzanne de. (ed). A finança capitalista. São Paulo: Alameda, 2010. p. 72-73).

Les rapports d'argent. $1^{\text {a }}$ ed. Paris : François Maspero, 1979. 
Marx a-ricardien: valeur, monnaie et prix au début du capital. In : Berthomieu, C. ; Cartelier, J. L. (Org). Ricardiens, keynésiens et marxistes : essais en economie politique non-néoclassique. Nice : Actes du Colloque de Nice, 1972

BUCI-GLAUCKSMANN, Christine. Sobre o conceito de crise do estado e sua história. In: Poulantzas, N. (Org). O estado em crise. Rio de Janeiro: Graal, 1985. p. 69.

CARDOSO, José Eduardo Martins. Curso de direito administrativo econômico. São Paulo: Malheiros, 2006. v. 3.

; QUEIROZ, João Eduardo Lopes; SANTOS, Márcia Walquíria Batista dos. (Org.). Curso de direito administrativo econômico. São Paulo: Malheiros, 2006. v. 2.

CHANG, Há-Joon. Chutando a escada: a estratégia do desenvolvimento em perspectiva histórica. São Paulo: Editora UNESP, 2004. p. 60-61).

CHESNAIS, François. A finança mundializada: raízes sociais e políticas, configuração, consequências. São Paulo: Boitempo, 2005.

. La mondialisation financière : genèse, coût et enjeux. Paris : Syros, 1996.

(Org). Mundialização: o capital financeiro no comando. In: Revista Outubro. N. 05. São Paulo: Instituto de Estudos Socialista, 2001.

DAVIS, Mike. 'Fordism' in crisis: a review of Michel Aglietta's Régulation et crises: l'expérience des etats-unis. In: JESSOP, B. Regulation theory and the crisis of capitalism: the parisian regulation school. Cheltenham: Edward Elgar, 2001. p. 120.

FAUSTO, Boris. História concisa do Brasil. São Paulo: Edusp, 2002. 
FILHO, Calixto Salomão. Regulação da atividade econômica: princípios e fundamentos. $2^{\mathrm{a}}$ ed. São Paulo: Malheiros, 2008.

FONTES, Virgínia. O Brasil e o capital-imperialismo: teoria e história. 3 ed. Rio de Janeiro: Editora UFRJ, 2010.

FUMAGALLI, Andrea; MEZZADRA, Sandro. A crise da economia global: mercados financeiros, lutas sociais e novos cenários políticos. $1^{\mathrm{a}}$ ed. Rio de Janeiro: Civilização Brasileira, 2011.

FURTADO, Celso. Interação entre decisões e estruturas. In: AGUIAR, R. F. (Org). Celso Furtado: essencial. São Paulo: Companhia das Letras, 2013. p. 145146

A comissão econômica para a América Latina. In: AGUIAR, R. F. (Org). Celso Furtado: essencial. São Paulo: Companhia das Letras, 2013. p. 105106.

GÉNÉREAU, Jacques. Introdução à política econômica. São Paulo: Loyola, 1995.

GILLY, Jean-Pierre ; Pecqueur, Bernard. La dimension locale de la regulation. In: BOYER, R.; SAILLARD, Y. (Org). Théorie de la régulation: l'état des savoirs. Paris: La Découvert, 2002. p. 309).

GRAMSCI, Antonio. Os intelectuais e a organização da cultura. São Paulo: Círculo do livro, 1982.

GRAU, Eros Roberto. Planejamento econômico e regra jurídica. São Paulo: Revista dos Tribunais, 1977.

A ordem econômica na Constituição de 1988. 11. ed. São Paulo: Malheiros, 2006. 
A empresa pública no Brasil: uma abordagem multidisciplinar. In: O controle político sobre as empresas públicas: objetivos, processos, extensão e conveniência. Brasília: IPEA/SEMOR, 1980. p. 205-58.

GUTTMANN, Robert. Monnaie et crédit dans la théorie de la régulation. In: BOYER, R.; SAILLARD, Y. (Org). Théorie de la régulation: l'état des savoirs. Paris: La Découvert, 2002. p. 85).

HARVEY, David. Para entender o capital. São Paulo:Boitempo, 2013. p. 303).

HILFERDING, Rudolf. O capitalismo financeiro. São Paulo: Nova Cultura, 1985. P. 40).

HIRSCH, Joachim. Observações teóricas sobre o Estado burguês e sua crise. In: Poulantzas, N. (Org). O estado em crise. Rio de Janeiro: Graal, 1985. p. 88).

Teoria materialista do estado: processos de transformação do sistema capitalista de estado. Rio de Janeiro: Revan, 2010.

HUSSON, Michel. Finança, hiperconcorrência e reprodução do capital. In: Brunhoff, Suzanne de. (ed). A finança capitalista. São Paulo: Alameda, 2010. p. 307-308).

IANNI, Octavio. Estado e planejamento econômico no Brasil. 2. ed. São Paulo: Civilização brasileira, 1977. (Coleção retratos do Brasil, 83).

JESSOP, Bob; SUM, Ngai-Ling. Beyond the regulation approach: putting capitalists economies in their places. Cheltenham: Edward Elgar, 2006.

El futuro del estado capitalista. Madrid: Catarata, 2008. 
. O estado e a construção de estados. Revista de Instituto de Estudos Socialistas, São Paulo: n. 15, p. 11-44, out. 2007.

Regulation theory and the crisis of capitalism: the parisian regulation school. Cheltenham: Edward Elgar, 2001.

. The capitalist state: Marxist theories and methods. Oxford: Martin Robertson, 1982.

JUILLARD, Michel. Régimes d'accumulation. In: BOYER, R.; SAILLARD, Y. (Org.). Théorie de la régulation: l'état des savoirs. Paris: La Découvert, 2002.

LACLAU, Ernesto. A razão populista. São Paulo: Três Estrelas, 2013

LEFF, Enrique. Ecologia, capital e cultura: a territorialização da racionalidade ambiental. Petrópolis: Vozes, 2009.

LÉNINE, Vladimir. Karl Marx. 2. ed. Pekin: Éditions du Peuple, 1970.

LÊNIN, Vladimir I. O imperialismo: fase superior do capitalismo. São Paulo: Global, 1985. p. 46.).

LEROY, Claude. Les salaires en longue période. In: BOYER, R.; SAILLARD, Y. (Org.). Théorie de la régulation: l'état des savoirs. Paris: La Découvert, 2002. p. 118 
LIPIETZ, Alain. Accumulation, crises, and ways out: some methodological reflections on the concept of regulation. In: JESSOP, B. Regulation theory and the crisis of capitalism: the Parisian regulation school. Cheltenham: Edward Elgar, 2001

. O capital e seu espaço. São Paulo: Nobel, 1988.

Alain. From althusserianism to "regulation theory". In: JESSOP, B. Regulation theory and the crisis of capitalism: the parisian regulation school. Cheltenham: Edward Elgar, 2001.

LORDON, Fréderic. Théorie de la régulation et politique économique. In: BOYER, R.; SAILLARD, Y. (Org). Théorie de la régulation: l'état des savoirs. Paris: La Découvert, 2002. p. 200.

MARX, Karl. As lutas de classe na França: de 1848 a 1850. 1 ed. São Paulo: Boitempo, 2012.

Do capital - Marx: vida e obra. São Paulo: Nova Cultural, 1999. (Coleção os pensadores). 2013.

O capital: crítica da economia política. Livro I. São Paulo: Boitempo,

Crítica da filosofia do direito de Hegel. São Paulo: Boitempo, 2005.

.Crítica do nacionalismo econômico. $1^{\text {a }}$ ed. Lisboa: Antígona, 2009. 1975.

Critique du programme de Gotha. 2. ed. Pequim: Editions du Peuple, . Manuscritos econômico-filosóficos. São Paulo: Martin Claret, 2002.

MASCARO, Alysson Leandro. Estado e forma política. São Paulo : Boitempo, 2013. p. 114-115. 2003.

Crítica da legalidade e do direito brasileiro. São Paulo: Quartier Latin, 
Atlas, 2003.

Filosofia do direito e filosofia política: a justiça é possível. São Paulo: Introdução ao estudo do direito. São Paulo: Quatier Latin, 2007.

Introdução à filosofia do direito: dos modernos aos contemporâneos. São Paulo: Atlas, 2002.

Lições de sociologia do direito. São Paulo: Quartier Latin, 2007.

NADEL, Henri. La régulation et Marx. In: BOYER, R.; SAILLARD, Y. (Org.). Théorie de la régulation: l'état des savoirs. Paris: La Découvert, 2002.

NAVES, Marcio Bilharinho. Marx: ciência e revolução. São Paulo: Moderna, 2000.

. Marxismo e direito: um estudo sobre Pachukanis. 1 ed. São Paulo: Boitempo, 2000. p. 72).

NUNES, António José Avelãs. Industrialização e desenvolvimento: a economia política do 'modelo brasileiro de desenvolvimento'. São Paulo: Quartier Latin, 2005.

ORLÉAN, André. Analyse économique des conventions. Paris : Presse Universitaires de France, 1994.

PASUKANIS, Evgeni B. Teoria geral do direito e marxismo. Rio de Janeiro: Renovar, 1989.

PAULANI, Leda Maria. A crise do regime de acumulação com dominância da valorização financeira e a situação do Brasil. Estudos Avançados, v. 23, n. 66, p. 25-39, 2009. 
POULANTZAS, Nicos. O Estado: o poder, o socialismo. 4 ed. São Paulo: Paz e Terra, 2000, 262.

RAYMOND, Pierre. Althusser philosophe. Paris: Presses Universitaires de France, 1997. (Collection actuel Marx confrontation).

ROUDINESCO, Élisabeth. Philosophes dans la tourmente. Paris: Fayard, 2005. (Collection histoire de la pensée).

SACHS, Ignacy. Desenvolvimento: includente, sustentável, sustentado. Rio de Janeiro: Garamond, 2008.

SINGER, Paul. Curso de introdução à economia política. $10^{\mathrm{a}}$ ed. Rio de Janeiro: Forense, 1986.

VEIGA, José Eli da. Desenvolvimento sustentável: o desafio do século XXI. 3. ed. Rio de Janeiro: Garamond, 2008.

WILliAMS, Eric. Capitalismo e escravidão. 1 ed. São Paulo: Companhia das Letras, 2012. 